\title{
Development of Polycaprolactone Microspheres with Controllable and Uniform Particle Size by Uniform Design Experiment in Emulsion Progress
}

\author{
Cheng-Tang Pan, ${ }^{1,2}$ Yeong-Maw Hwang, ${ }^{1}$ Yu-Min Lin, ${ }^{1}$ \\ Song-Wei Zeng, ${ }^{1}$ Shao-Yu Wang, ${ }^{1}$ Shiao-Wei Kuo, ${ }^{3}$ Shin-Pon Ju, ${ }^{1}$ \\ Shih-Shin Liang, ${ }^{4}$ Zong-Hsin Liu, ${ }^{5}$ and Chung-Kun Yen ${ }^{1 *}$ \\ ${ }^{1}$ Department of Mechanical and Electro-Mechanical Engineering, National Sun Yat-sen University, \\ Kaohsiung 80424, Taiwan \\ ${ }^{2}$ Institute of Medical Science and Technology, National Sun Yat-sen University, Kaohsiung 80424, Taiwan \\ ${ }^{3}$ Department of Materials and Optoelectronic Science, National Sun Yat-sen University, Kaohsiung 80424, Taiwan \\ ${ }^{4}$ Department of Biotechnology, Kaohsiung Medical University, Kaohsiung 80424, Taiwan \\ ${ }^{5}$ Metal Industries Research \& Development Centre, Kaohsiung 80424, Taiwan
}

(Received May 31, 2018; accepted October 11, 2018)

Keywords: polycaprolactone, emulsification, uniform design experiment, spherical carrier

In this study, we propose a method of manufacturing spherical carriers with controllable and uniform particle size. Emulsification and a uniform design experiment were carried out to obtain the optimal fabrication process. The results show that a stable emulsification of oil in water $(\mathrm{O} / \mathrm{W})$ was achieved with an $\sim 18.18 \mathrm{wt} \%$ polycaprolactone $(\mathrm{PCL})$ oil solution and an $\sim 13.02 \mathrm{wt} \%$ polyvinyl alcohol (PVA) aqueous solution. Finally, the uniformity of the PCL microcarriers was $\sim 87.9 \%$ with a particle size range of $10-20 \mu \mathrm{m}$. In the future, medical carriers will be loaded with different officially approved medicines. The carriers could improve the bioavailability of pharmaceutical products, extend the duration of their effectivity due to drug and minimize their side effects.

\section{Introduction}

Research on nanotechnology for medical applications has been proceeding for decades. With the strength of industry and research institutes, a number of nanotechnology platforms were established in relation to applications. Polycaprolactone (PCL) is an aliphatic polyester that has been intensively investigated as a potential biomaterial. Although it was recognized that PCL can be degraded by microorganisms, which led to the evaluation of PCL as a biodegradable packaging material, PCL can also be degraded by a hydrolytic mechanism under physiological conditions. ${ }^{(1)}$ Tokiwa and Suzuki discussed the hydrolysis of PCL and the biodegradation of PCL by fungi, and showed that PCL was degraded enzymatically. ${ }^{(2)}$ PCL is highly processible since it is soluble in a wide range of organic solvents, and it has a low melting point $\left(\sim 55-60^{\circ} \mathrm{C}\right)$ and glass transition temperature $\left(\sim 60^{\circ} \mathrm{C}\right)$ while having the capability to form

*Corresponding author: e-mail: alden0113@gmail.com https://doi.org/10.18494/SAM.2019.2094 
miscible blends with a wide range of polymers. ${ }^{(3)}$ PCL microspheres were prepared by several different methods, some of which were reviewed by Freiberg and Zhu. ${ }^{(4)}$ Spherical carriers are formed using oil-soluble organic monomers and dispersed in aqueous media (oil in water, $\mathrm{O} / \mathrm{W}$ ) or formed by water-soluble monomers dissolved in water and dispersed in an organic medium (water in oil, W/O). ${ }^{(5)}$ The polymerization of dispersed monomers is achievable by various methods including emulsion, suspension, and dispersion techniques. ${ }^{(6)}$ The carriers were employed for medical applications, and their size uniformity is very important for a drug, because the size of the microspheres affects the amount of drug loaded in the carrier. Spherical size and size distribution are reproducible but often poorly controllable. Standard deviations of $25-50 \%$ of the mean diameter were not uncommon. Furthermore, precise size control may lead to advanced delivery systems not possible for polydisperse spheres. ${ }^{(7-12)}$ Uniform microspheres of approximately 1-5 $\mu \mathrm{m}$ diameters would be ideal for the passive targeting of professional antigen-presenting cells such as macrophages and dendritic cells. ${ }^{(13,14)}$ Similarly, microspheres of $10-20 \mu \mathrm{m}$ diameters could be used to target the tortuous capillary bed of tumor tissues by chemoembolization. ${ }^{(15)}$

Therefore, in this study, we present a method of manufacturing spherical carriers with controllable and uniform particle size. Through emulsification and a uniform design experiment, we obtained the optimal fabrication process and the precise microsphere fabrication system.

\section{Experimental Methods}

A uniform design experiment method and emulsification were carried out to obtain the optimum uniform particle size of the microspheres. Emulsification was conducted by dripping the oil phase (PCL) into the water phase [polyvinyl alcohol (PVA)] to fabricate microspheres. Variable parameters, which were factors in the uniform experiment method, were considered, such as the speed of PVA solution stirring (magnetic stirring), PVA solution concentration, PCL solution concentration, the rate of PCL solution flow by an infusion pump, and the distance between the PCL droplets and the PVA solution. The experimental processes are schematically shown in Fig. 1.

\subsection{Experimental processes and material properties}

First, the proportion of PCL with ethyl acetate (EA) was selected, and they were placed in an ultrasonic oscillator with shaking for 45 min to obtain the PCL solution. The PCL solution was placed in a syringe $(5 \mathrm{ml}$ syringe with a Teflon tube and a tip needle with a red needle, the inside diameter of which was $0.25 \mathrm{~mm}$ ), and the syringe was fixed on the feeding injection pump for injection and sensors. The time of dripping into the droplets was about $4 \mathrm{~s}$. On the other hand, the proportion of PVA was selected, and the PVA solution was placed on the electromagnetic heating stirrer with a magnetic stone bar at different stirring speeds. Figure 2 schematically shows the PCL and PVA emulsification processes. Figure 3 shows the equipment used, including the infusion pump, height gage, hot plate, needle, and collection region. 


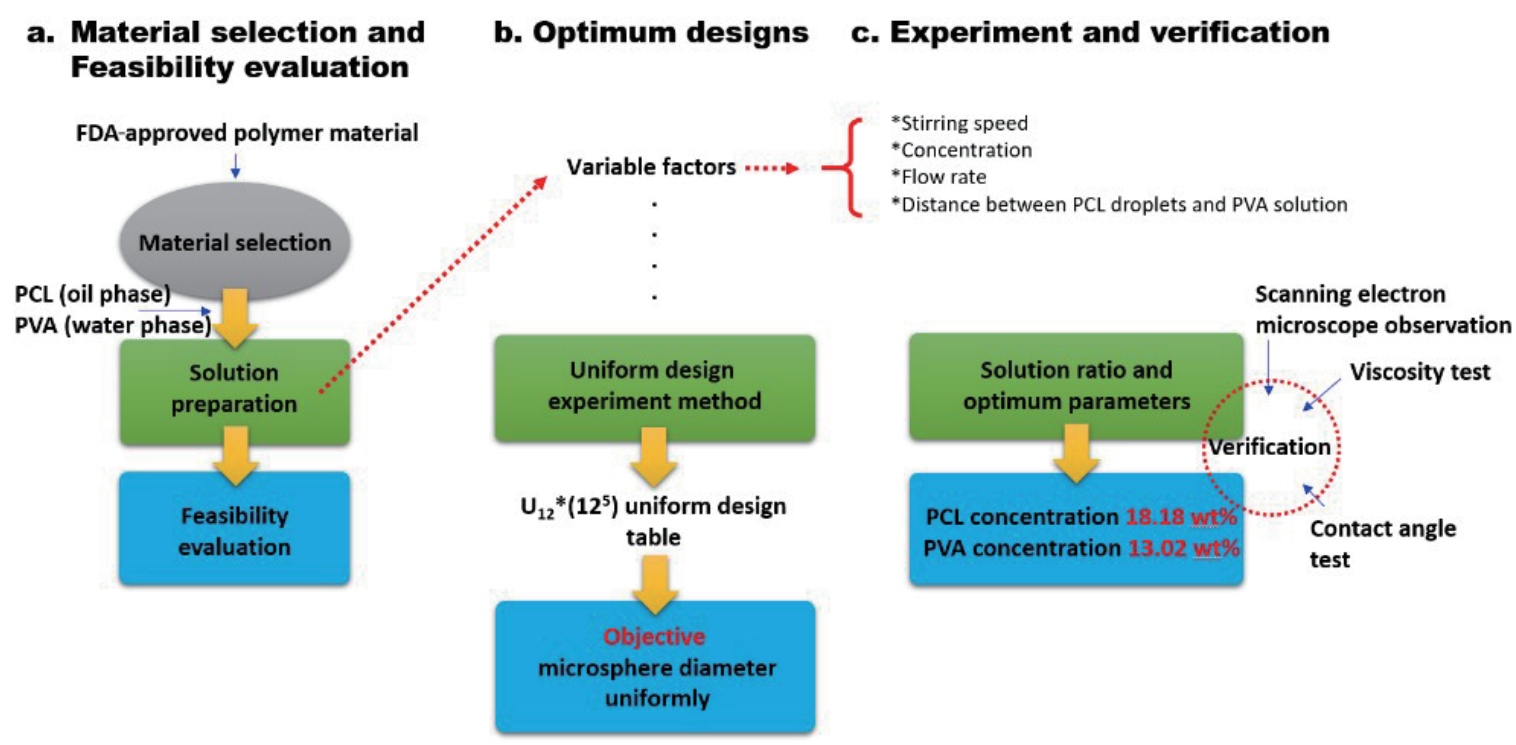

Fig. 1. (Color online) Illustration of the experiment and processes.

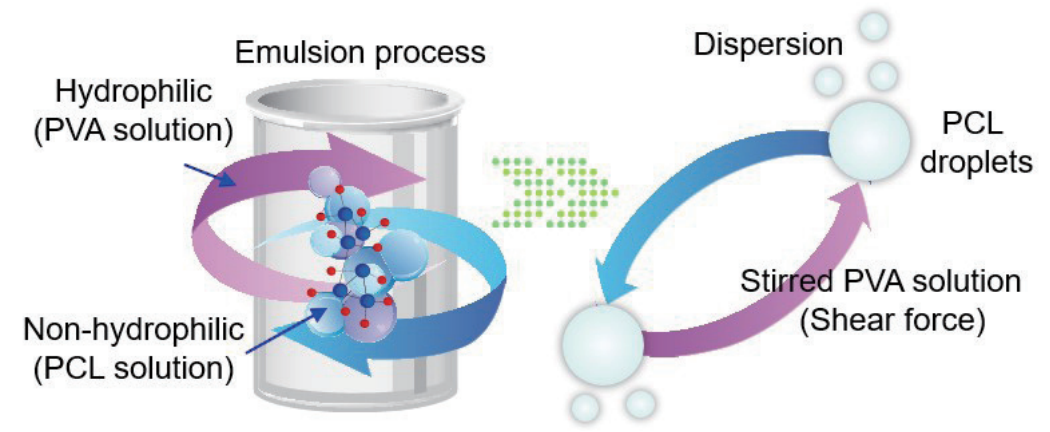

Fig. 2. (Color online) Schematic emulsion of PCL and PVA solutions.

The surface morphology and particle size of the microcarriers were strongly affected by the solution in this process, which were adjusted by using different concentrations of the solution and instrument parameters. The preparation steps of the PCL solution are as follows. The PCL solution was dropped into the aqueous solution. Owing to the variable stirring speed of the magnetic stirrer, PCL precipitated from the organic solvent and precipitates attached to each other. Therefore, the original aqueous solution was replaced by a water-soluble polymer material, PVA. The use of PVA, which is not soluble in general organic solvents, reduced the stickiness of the PCL carriers in the emulsion. The main materials were PCL (weight average molecular weight $\mathrm{Mw}$ of $\sim 14000$ and number average molecular weight Mn of $\sim 10000$ ), PVA (Mw of $30000-70000$ ), and EA as the solvent. The oil solution was prepared by dissolving PCL in EA, and the aqueous solution was prepared by dissolving PVA in deionized water. Then, the oil solution was dropped into the aqueous solution to form the emulsion type of $\mathrm{O} / \mathrm{W}$. 


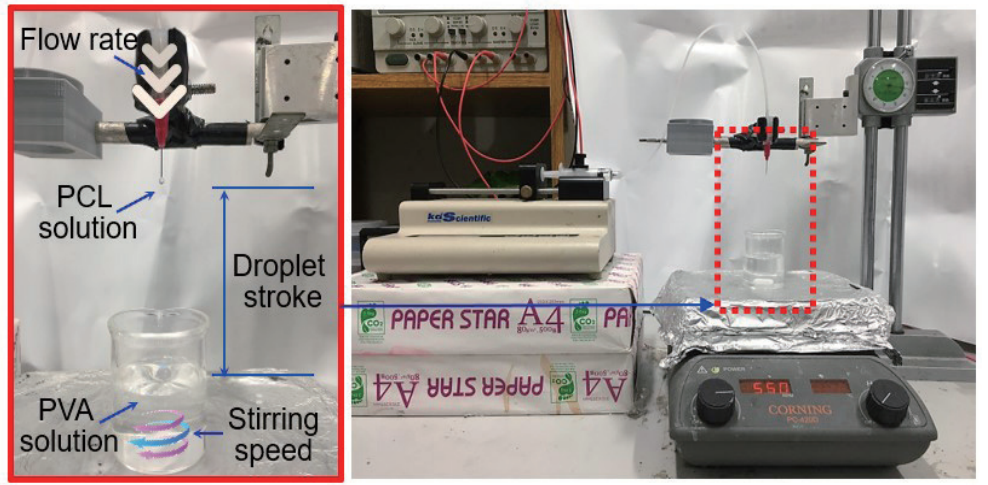

Fig. 3. (Color online) Photos of equipment.

\subsection{Uniform design experiment method}

In this experiment, we used the uniform design experimental method, which reduced effectively the number of experiments, and spread the experimental points uniformly within the overall experimental range, so as to effectively perform regression analysis and optimization. This method can reduce the experimental cost, improve product quality and effectiveness, and achieve higher economic benefits. Therefore, the objective of this experiment was to study the percentage of microsphere diameter uniformity after PCL emulsification. Thus, the percentage of microsphere size uniformity was estimated to determine the impact of different factors on the process. The $\mathrm{U}_{12} *\left(12^{5}\right)$ uniform experimental design was used to layout 5 parameters in this study, namely, the effects of stirring speed, PVA concentration, PCL concentration, flow rate, and height on the percentage of microsphere diameter uniformity of PCL carriers. In order to facilitate the follow-up performance and observation, the factors were represented by the codes A1-A5.

The designs are shown in Table 1. It includes the required factor levels (Table 2) and the uniform experimental design process parameters with the $\mathrm{U}_{12} *\left(12^{5}\right)$ uniform experimental design. Finally, a suite of software packages optimized using the MATLAB analysis software was used as a solver. The best combination of parameters was obtained from the established Kriging model. The highest percentage of microsphere uniformity obtained by the PCL microsphere process was set as the target of the Kriging model. The best microsphere diameter process data were then calculated.

\section{Results and Discussion}

\subsection{Experiment results of U1-U12 levels}

The experimental results show that the low concentration of the PVA solution, resulting in incomplete formation of PCL thin shell, and the immature carriers existed in sheet materials 
Table 1

Factor number description and design factor limits of uniform design method.

\begin{tabular}{lccc}
\hline Process parameters & $\begin{array}{c}\text { Factor number } \\
\text { description }\end{array}$ & Minimum & Maximum \\
\hline Stirring speed & A1 & $250 \mathrm{rpm}$ & $800 \mathrm{rpm}$ \\
PVA solution concentration & A2 & $2 \mathrm{wt} \%$ & $24 \mathrm{wt} \%$ \\
PCL solution concentration & A3 & $14 \mathrm{wt} \%$ & $36 \mathrm{wt} \%$ \\
Flow rate & A4 & $0.05 \mathrm{ml} / \mathrm{hr}$ & $0.5 \mathrm{ml} / \mathrm{hr}$ \\
Height & A5 & $5 \mathrm{~mm}$ & $60 \mathrm{~mm}$ \\
\hline
\end{tabular}

Table 2

Levels and factors of uniform design method.

\begin{tabular}{lccccc}
\hline Level number & $\begin{array}{c}\mathrm{A} 1 \\
\text { (rpm) }\end{array}$ & $\begin{array}{c}\mathrm{A} 2 \\
(\mathrm{wt} \%)\end{array}$ & $\begin{array}{c}\mathrm{A} 3 \\
(\mathrm{wt} \%)\end{array}$ & $\begin{array}{c}\mathrm{A} 4 \\
(\mathrm{ml} / \mathrm{h})\end{array}$ & $\begin{array}{c}\mathrm{A} 5 \\
(\mathrm{~mm})\end{array}$ \\
\hline Level 1 (U1) & 250 & 2 & 14 & 0.05 & 5 \\
Level 2 (U2) & 300 & 4 & 16 & 0.1 & 10 \\
Level 3 (U3) & 350 & 6 & 18 & 0.15 & 15 \\
Level 4 (U4) & 400 & 8 & 20 & 0.2 & 20 \\
Level 5 (U5) & 450 & 10 & 22 & 0.25 & 25 \\
Level 6 (U6) & 500 & 12 & 24 & 0.3 & 30 \\
Level 7 (U7) & 550 & 14 & 26 & 0.35 & 35 \\
Level 8 (U8) & 600 & 16 & 28 & 0.4 & 40 \\
Level 9 (U9) & 650 & 18 & 30 & 0.45 & 45 \\
Level 10 (U10) & 700 & 20 & 32 & 0.5 & 50 \\
Level 11 (U11) & 750 & 22 & 34 & 0.55 & 55 \\
Level 12 (U12) & 800 & 24 & 36 & 0.6 & 60 \\
\hline
\end{tabular}
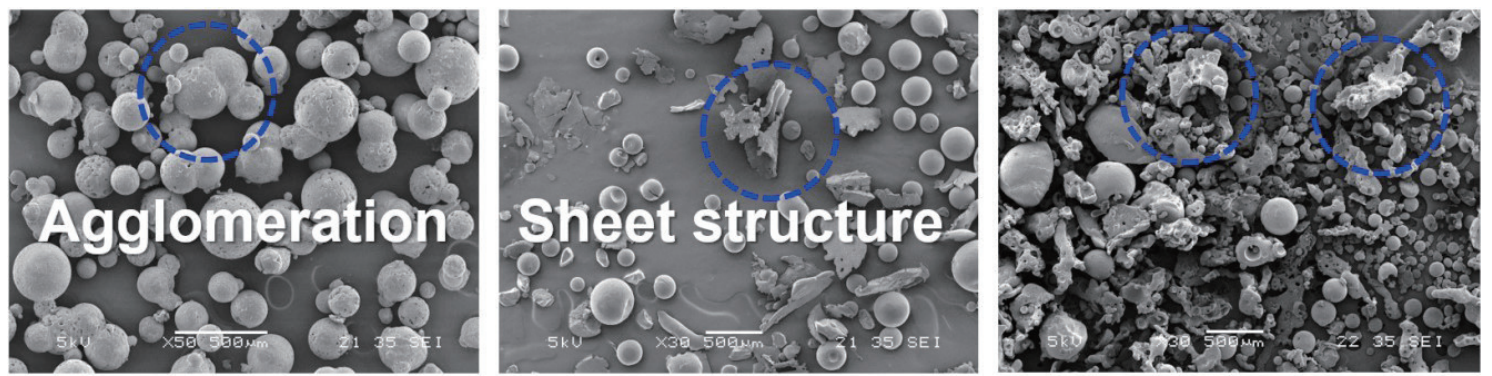

Fig. 4. (Color online) PCL microsphere formation mechanism under low PVA concentration.

under a strong shearing force during the mixing process, as shown in Fig. 4. If the stirring speed and PVA viscosity were increased, owing to the high viscosity of the PVA solution, which was not stirred smoothly, the organic solvent was not successfully precipitated from the PCL, and the PCL carriers became rarer and the particle size larger, as shown in Fig. 5. From the result of linear regression analysis, the parameters with the greatest effects were calculated using the degree of contribution in the analysis of variation (ANOVA), and the concentration of the PCL solution was obtained, which was $63 \%$, followed by the stirring speed and the PVA concentration, which were $690 \mathrm{rpm}$ and $4.16 \%$, respectively. 


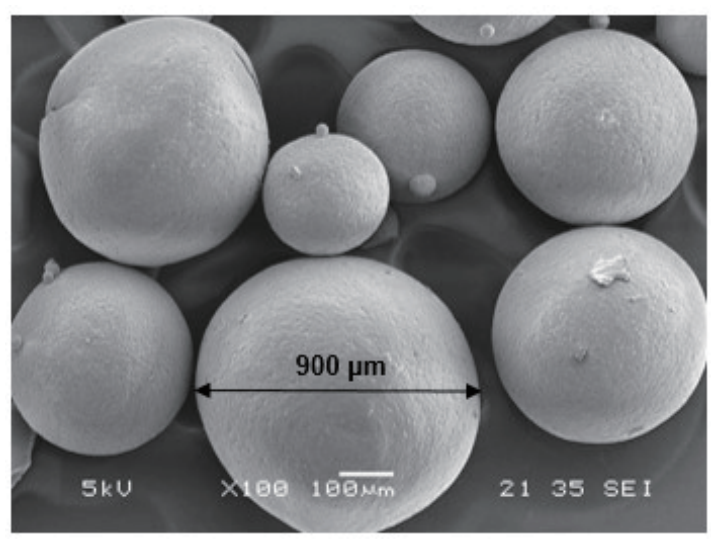

Fig. 5. A high concentration of PVA caused the PCL carriers to become rarer and the particles larger.

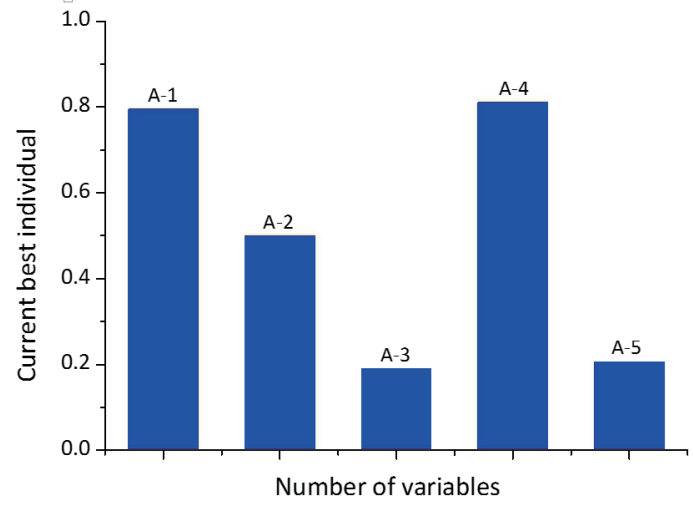

Fig. 6. (Color online) Normalization of parameters.
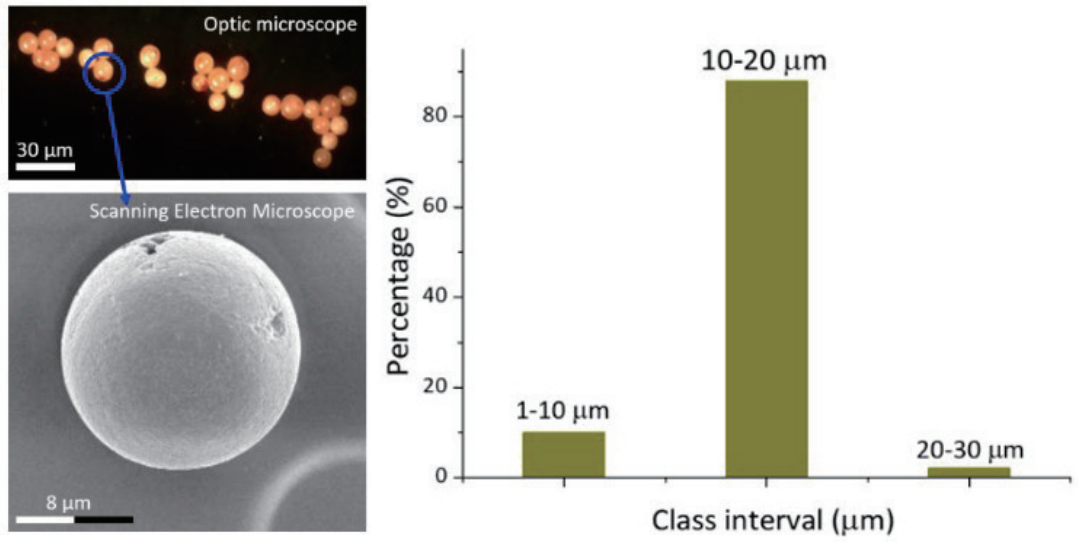

Fig. 7. (Color online) Microsphere diameter uniformity and optimization analysis.

\subsection{Results of optimization module}

Through the genetic algorithm (GA) function module in MATLAB, which was used to solve and verify the optimal experimental parameters in subsequent experiments, the uniform experimental design made the optimal uniformity of PCL microsphere diameter as the target function to normalize the best combination of factors. Figure 6 shows that the parameters in the manufacturing process include the rotational speed of $690 \mathrm{rpm}$, the PVA concentration of $13.02 \mathrm{wt} \%$, the PCL concentration of $18.18 \mathrm{wt} \%$, the pump feeding rate of $0.5 \mathrm{ml} / \mathrm{h}$, and the PCL solution drop height of $16.33 \mathrm{~mm}$. After the experimental process, the particle size range was about $10-20 \mu \mathrm{m}$ and the uniformity was about $87.9 \%$, as determined by scanning electron microscopy (SEM) and optical microscopy, as shown in Fig. 7. 


\subsection{Solution analysis of optimization results}

The optimization level combination parameter prediction and experiment results were compared. An error of about $9.4 \%$ was observed between the results. A rheometer and a contact angle measurement analyzer were further used to analyze the physical properties of the polymer solution in the case of using $18.18 \mathrm{wt} \%$ PCL solution. Owing to the fast evaporation of EA during the process, data were collected once per second to reduce the distortion of the rheological properties of the PCL solution. The results showed that the best parameter of the PCL solution was a viscosity of $15.12 \mathrm{cP}$. As shown in Fig. 8, the shear strain rate and the shear stress of the PCL solution showed a positive linear relationship, which is similarly observed in a Newtonian fluid. In instrument measurement, the contact angle of the PCL solution was $83.75^{\circ}$, which was close to $90^{\circ}$; this means that the solution is hydrophobic, as shown in Fig. 9 .

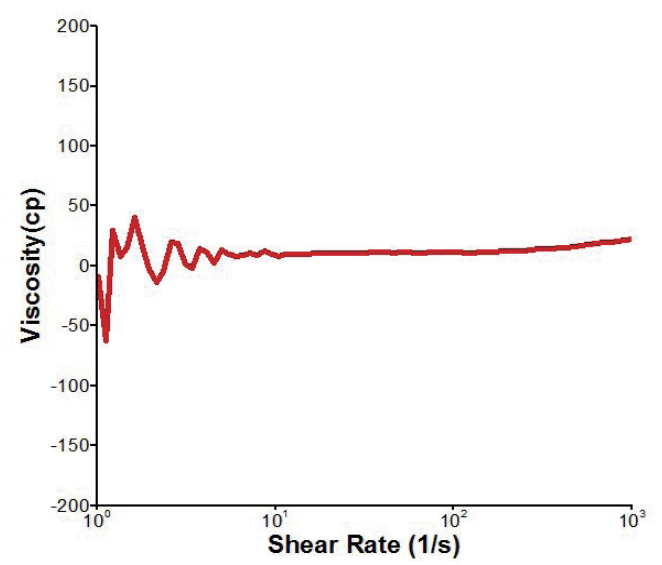

(a)

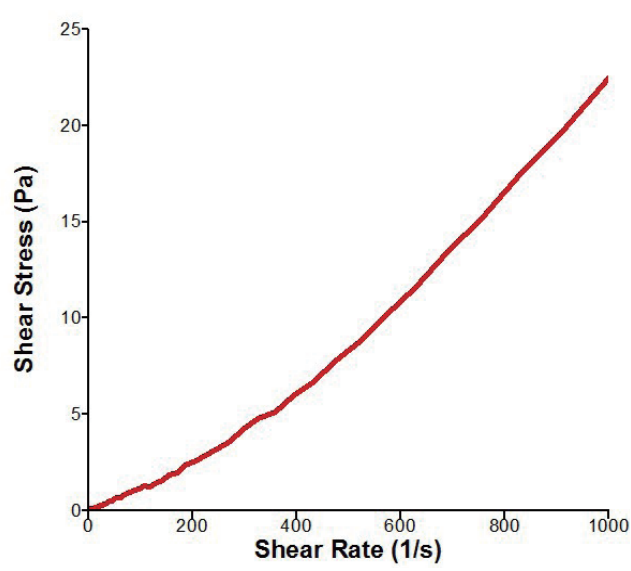

(b)

Fig. 8. (Color online) Rheometer analysis results of optimization solution: (a) shear rate and viscosity relationship and (b) shear rate and shear stress relationship.

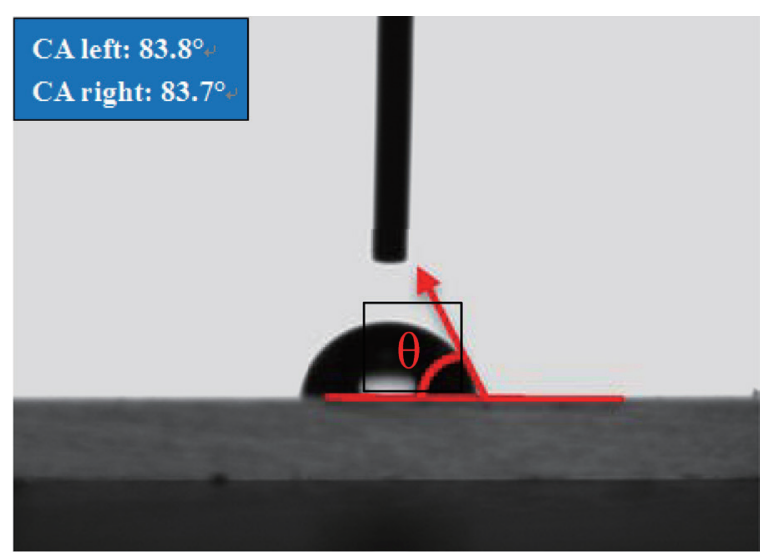

Fig. 9. (Color online) Contact angle of optimization solution. 


\section{Conclusions}

In the experiment, a PVA aqueous solution was prepared to have a hydrophobic end to prevent the PCL microspheres from sticking together during the processing. According to the uniform design experimentation with regression analysis, the parameters were as follows: rotational speed of $690 \mathrm{rpm}$, PVA concentration of $13.02 \mathrm{wt} \%$, PCL concentration of 18.18 wt\%, pump feeding rate of $0.5 \mathrm{ml} / \mathrm{h}$, and PCL solution drop height of $16.33 \mathrm{~mm}$. The PCL microspheres achieved $\sim 10-20 \mu \mathrm{m}$ diameter and $87.9 \%$ uniformity. Using the rheometer and contact angle measurement analyzer, we further analyzed the physical properties of the 18.18 wt \% PCL solution. The results showed that the best parameter for the PCL solution was a viscosity of $15.12 \mathrm{cP}$. The shear strain rate and the shear stress for the PCL solution showed a positive relationship, which is similarly observed in a Newtonian fluid. The contact angle of the PCL solution was $83.75^{\circ}$, which was close to $90^{\circ}$; this means that its surface is approximately hydrophobic. In the future, biodegradable microcarriers will be loaded with different approved medicines on the market. In drug delivery systems, biodegradable microcarriers can provide drugs at the needed amount and at the right time to the right location in the body and minimize side effects.

\section{References}

1 T. Hayashi: Prog. Polym. Sci. 19 (1994) 663.

2 R. Chandra and R. Rustgi: Prog. Polym. Sci. 23 (1998) 1273.

3 L. S. Nair and C. T. Laurencin: Prog. Polym. Sci. 32 (2007) 762.

4 S. Freiberg and X. Zhu: Int. J. Pharm. 282 (2004) 1.

5 F. Candau: Encyclopedia of Polymer Science and Engineering (Wiley, New York, 1985) 2nd ed., p. 719.

6 I. Piirma: Encyclopedia of Polymer Science and Engineering (Wiley, New York, 1985) 2nd ed., p. 125.

7 J. M. Weissman, H. B. Sunkara, A. S. Tee, and S. A. Asher: Science 274 (1996) 959.

8 R. Langer and N. Peppas: J. Macromol. Sci., Part C 23 (1983) 61.

9 R. Langer: Science 249 (1990) 1527.

10 A. Gpferich and R. Langer: Eur. J. Pharm. Biopharm. 41 (1995) 81.

11 Y.-F. Maa and C. Hsu: J. Microencapsulation 13 (1996) 53.

12 M. A. Tracy: Biotechnol. Progr. 14 (1998) 108.

13 C. Evora, I. Soriano, R. A. Rogers, K. M. Shakesheff, J. Hanes, and R. Langer: J. Controlled Release 51 (1998) 143.

14 S. Akhtar and K. J. Lewis: Int. J. Pharm. 151 (1997) 57.

15 C. R. Dass and M. A. Burton: Drug Delivery 6 (1999) 243. 